OPEN ACCESS

Edited by:

Marco Scarpa,

University Hospital of Padua, Italy

Reviewed by:

Ping Li,

Fujian Medical University, China

Jun Lyu,

First Affiliated Hospital of Xi'an

Jiaotong University, China

*Correspondence:

Shengping L

lishengp@mail.sysu.edu.cn

${ }^{\dagger}$ These authors have contributed equally to this work

Specialty section:

This article was submitted to Surgical Oncology,

a section of the journal

Frontiers in Oncology

Received: 06 March 2020 Accepted: 31 March 2020 Published: 28 April 2020

Citation:

He C, Sun S, Zhang Y, Lin X and Li S

(2020) Score for the Overall Survival Probability of Patients With Pancreatic Adenocarcinoma of the Body and Tail

After Surgery: A Novel

Nomogram-Based Risk Assessment.

Front. Oncol. 10:590

doi: 10.3389/fonc.2020.00590

\section{Score for the Overall Survival Probability of Patients With Pancreatic Adenocarcinoma of the Body and Tail After Surgery: A Novel Nomogram-Based Risk Assessment}

\author{
Chaobin $\mathrm{He}^{1+}$, Shuxin Sun ${ }^{1+}$, Yu Zhang ${ }^{2 \dagger}$, Xiaojun $\mathrm{Lin}^{1}$ and Shengping $\mathrm{Li}^{1 *}$ \\ ${ }^{1}$ State Key Laboratory of Oncology in South China, Department of Pancreatobiliary Surgery, Collaborative Innovation Center \\ for Cancer Medicine, Sun Yat-sen University Cancer Center, Guangzhou, China, ${ }^{2}$ State Key Laboratory of Ophthalmology, \\ Retina Division, Zhongshan Ophthalmic Center, Sun Yet-sen University, Guangzhou, China
}

Pancreatic adenocarcinoma of the body and tail often has a dismal prognosis and lacks a specific prognostic stage. The aim of this study was to construct a nomogram for predicting survival of patients with pancreatic adenocarcinoma of the body and tail after surgery. Data of patients were selected from the Surveillance, Epidemiology, and End Results (SEER) database and from medical records of Sun Yat-sen University Cancer Center (SYSUCC). In a multivariate analysis for overall survival (OS), the following six variables were identified as independent predictors and incorporated into the nomogram: age, tumor differentiation, tumor size, lymph node ratio $(L N R)$, and chemotherapy. A nomogram was built based on independent risk predictors. The concordance index (C-index) for nomogram, Tumor-Node-Metastasis (TNM) 7th and 8th stage system were 0.775 [95\% confidence interval $(\mathrm{Cl}), 0.731-0.819], 0.617(95 \% \mathrm{Cl}, 0.575-0.659)$, and $0.632(95 \% \mathrm{Cl}, 0.588-0.676)$, respectively. The calibrated nomogram predicted survival rates which closely corresponded to the actual survival rates. Furthermore, the values of the area under receiver operating characteristic (ROC) curves (AUC) of the nomograms were higher than those of the TNM 7th or 8th stage system in predicting 1-, 2-, and 3-year survival of patients in training and external validation cohorts. The well-calibrated nomogram could be used to predict prognosis for patients with pancreatic adenocarcinoma of the body and tail after surgery.

Keywords: pancreatic adenocarcinoma, nomogram, overall survival, prognosis, SEER

\section{INTRODUCTION}

Pancreatic ductal adenocarcinoma (PDAC), which represents one of the most common gastrointestinal tumors, is the fourth cause of cancer deaths in developed countries (1). Surgery leads to the best chance of survival with a 5-year overall survival (OS) rate of only 5-7\% (2). Compared with PDAC of the head of the pancreas, the discrepancies of ontogeny would lead to significant differences in cell composition, blood supply, lymphatic and venous backflow in PDAC of the body and tail of the pancreas (3). Moreover, due to 
the lack of obstructive jaundice, PDAC which occurred at the body and tail has a lower resectable rate and a more dismal prognosis than PDAC of the head $(4,5)$.

Several independent prognostic factors of PDAC have been identified, such as lymph node (LN) metastasis, tumor size, and resection margin $(6,7)$. In these studies, the prognostic influence of risk factors on PDAC of the body and tail is only estimated because the reported predictors of prognosis mainly focus on PDAC occurring at the pancreatic head. It is known that the differences in ontogeny lead to significant differences in clinical characteristics between tumors occurred at the head or the body/tail (8). However, the 8th edition of the tumor-node-metastasis (TNM) staging system of the American Joint Commission on Cancer (AJCC), (9) which is commonly used to stage diseases, takes only common predictive factors into account and does not incorporate the tumor site. Furthermore, the 8th edition of the TNM is still cumbersome and not specifically designed to predict prognosis. The TNM staging system algorithm, which uses risk factors to make clinical decisions, needs to be validated. The lack of an accurate and reliable staging system for PDAC of the body and tail makes it difficult and challenging for doctors to appropriately identify patients at risk of long-term survival. Therefore, it is necessary to develop a technically feasible and an easily accessible clinical staging system to stratify the prognosis of patients with PDAC of the body and tail when surgery is urgently required.

Nomograms, which have been adopted in various cancers (10-13) and have shown favorable results, compared with traditional TNM staging systems, (14) are simple graphical depictions of the predictive model and are used to provide the probabilities of outcomes for individual patient (15). However, few studies have reported specific nomograms for patients with PDAC of the body and tail after surgery. The purpose of this analysis was to develop a clinically useful nomogram which could be used to predict the prognosis of patients with PDAC of the body and tail after surgery.

\section{MATERIALS AND METHODS}

\section{Study Design}

In this retrospective study, according to the inclusion and exclusion criteria, suitable patients were selected and clinicopathological variables of these patients were retrospectively reviewed. Extensive evaluation of concerning risk factors were conducted using a univariate analysis for all included variables and a multivariate analysis was adopted to select the independent risk factors. A nomogram was built based on these independent risk factors and validated in both training and external validation cohorts. The predictive power of the established nomogram was also compared with that of the 7th and 8th editions of TNM stage systems. This study was approved by the Institutional Review Board (IRB) of the Sun Yat-sen University Cancer Center. Each individual participant from the SYSUCC database provided informed written consent. All procedures performed in studies involving human participants were in accordance with the 1964 Helsinki Declaration and its later amendments or comparable ethical standards.

\section{Patients}

The Surveillance, Epidemiology, and End Results (SEER) program maintains the largest clinical dataset in the United States and provides data on cancer incidence and survival. For this research, the training cohort of patients with PDAC of the body and tail were obtained from the SEER database (2004-2015). The International Classification of Diseases for Oncology, Third Edition (ICD-O-3), histology code 8140/3 and site codes C25.1 and C25.2, were followed in the SEER database using SEER*Stat software version 8.3.4. The second cohort of patients was obtained from the SYSUCC (2009-2017), which was used as an external validation cohort. The inclusion criteria were as follows: (1) pathologically confirmed pancreatic adenocarcinoma and radiologically confirmed PDAC of the body and tail; (2)

\section{SEER cohort}

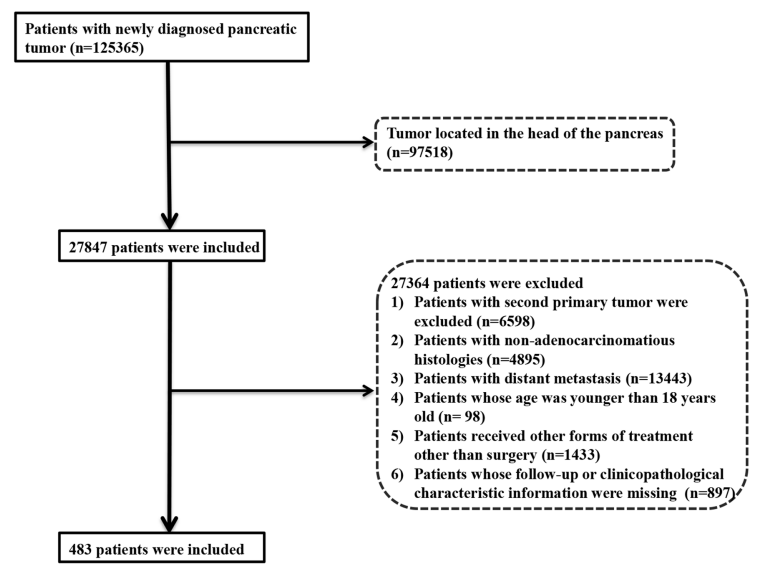

\section{SYSUCC cohort}

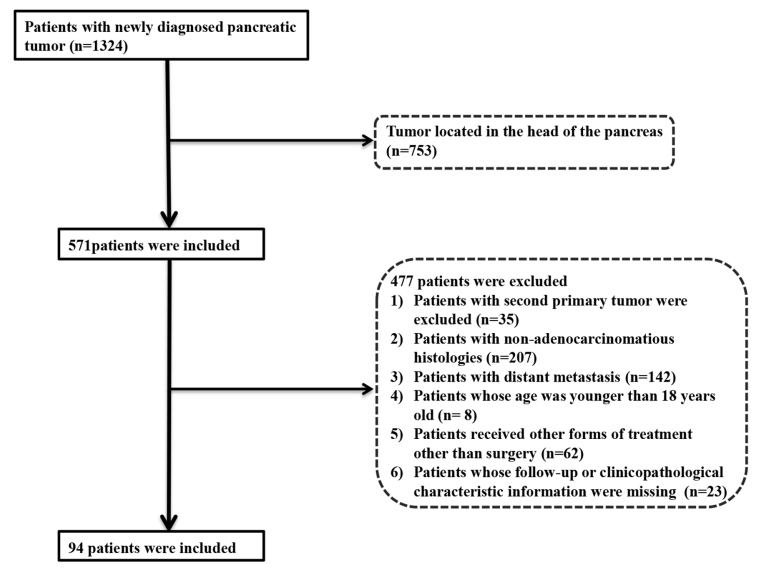

FIGURE 1 | The flow diagram of the selection process for the study cohort. 
radical surgical resection. The following exclusion criteria were adopted: (1) second primary cancer; (2) distant metastases; (3) other treatments only, including chemotherapy or radiotherapy; and (4) missing or incomplete information. The information of TNM system was adopted in this study, which was in accordance with previous studies $(16,17)$.

\section{Data Collection}

Pathological and clinical variables, such as age at diagnosis, gender, tumor size, tumor differentiation, TNM stage, chemotherapy, radiotherapy, and follow-up information, were extracted from the SEER and SYSUCC databases. Sixty was used as the cutoff value of age in this study. The time-dependent receiver operating characteristic (ROC) curve analysis was used to determine the optimal cutoff value for lymph node ratio (LNR), which was defined as the same as previous studies (18).
OS was calculated from the date of surgery to the date of death or last follow-up.

\section{Statistical Analysis}

The chi-square test and Fisher's exact test were used to compare categorical data, which are shown as frequencies and proportions. Variables that were significantly associated with OS were analyzed in the multivariate analysis using the Cox regression model to determine the independent predictive factors, along with the corresponding 95\% confidence interval (CI). Survival differences were compared with log-rank test. The nomogram was developed according to previous protocols (18-20) and the area under the ROC curve (AUC) was used to evaluate and compare the precision of predicting 1-, 2-, and 3-year survival with the nomogram. All statistical analyses were performed using $\mathrm{R}$ version 3.4.2 software (The $\mathrm{R}$ Foundation for Statistical

TABLE 1 | Characteristics and overall survival of patients with pancreatic adenocarcinoma of the body and tail.

\begin{tabular}{|c|c|c|c|c|c|c|c|c|c|c|c|}
\hline \multicolumn{2}{|c|}{ Characteristics } & \multicolumn{5}{|c|}{ SEER cohort } & \multicolumn{5}{|c|}{ SYSUCC cohort } \\
\hline & & \multirow[t]{2}{*}{ Patients } & & \multicolumn{2}{|c|}{ Tumor site } & \multirow[t]{2}{*}{$P$} & \multirow[t]{2}{*}{ Patients } & & \multicolumn{2}{|c|}{ Tumor site } & \multirow[t]{2}{*}{$P$} \\
\hline & & & & Body & Tail & & & & Body & Tail & \\
\hline Total & & 483 & 100.0 & 213 & 270 & & 94 & 100.0 & 41 & 53 & \\
\hline \multirow[t]{2}{*}{ Age } & $<60$ years & 318 & 65.8 & 137 & 181 & 0.563 & 72 & 76.6 & 29 & 43 & 0.326 \\
\hline & $\geq 60$ years & 165 & 34.2 & 76 & 89 & & 22 & 23.4 & 12 & 10 & \\
\hline \multirow[t]{2}{*}{ Gender } & Male & 238 & 49.3 & 106 & 132 & 0.855 & 52 & 55.3 & 20 & 32 & 0.300 \\
\hline & Female & 245 & 50.7 & 107 & 138 & & 42 & 44.7 & 21 & 21 & \\
\hline \multirow[t]{3}{*}{ Race } & White & 378 & 78.3 & 177 & 201 & 0.052 & & & & & NA \\
\hline & Black & 57 & 11.8 & 17 & 40 & & & & & & \\
\hline & Asian & 48 & 9.9 & 20 & 28 & & & & & & \\
\hline \multirow[t]{3}{*}{ Tumor size } & $\leq 2 \mathrm{~cm}$ & 76 & 15.7 & 37 & 39 & 0.107 & 13 & 13.8 & 3 & 10 & 0.274 \\
\hline & $2 \sim 4 \mathrm{~cm}$ & 185 & 38.3 & 90 & 95 & & 28 & 29.8 & 13 & 15 & \\
\hline & $>4 \mathrm{~cm}$ & 222 & 46.0 & 87 & 135 & & 53 & 56.4 & 25 & 28 & \\
\hline \multirow[t]{3}{*}{ Tumor differentiation } & Well & 47 & 9.7 & 18 & 29 & 0.510 & 6 & 6.4 & 3 & 3 & 0.948 \\
\hline & Moderately & 242 & 50.1 & 98 & 144 & & 81 & 86.2 & 35 & 46 & \\
\hline & Poorly & 194 & 40.2 & 89 & 105 & & 7 & 7.4 & 3 & 4 & \\
\hline \multirow[t]{2}{*}{ LN metastasis (7th edition) } & Absent & 231 & 47.8 & 120 & 111 & 0.002 & 86 & 91.4 & 37 & 49 & 0.725 \\
\hline & Present & 252 & 52.2 & 99 & 159 & & 8 & 8.6 & 4 & 4 & \\
\hline \multirow[t]{3}{*}{ LN metastasis (8th edition) } & Absent & 231 & 47.8 & 120 & 111 & 0.006 & 86 & 91.4 & 37 & 49 & 0.930 \\
\hline & $1 \sim 3$ LNs & 180 & 37.3 & 65 & 115 & & 4 & 4.3 & 2 & 2 & \\
\hline & $\geq 4 \mathrm{LNs}$ & 72 & 14.9 & 28 & 44 & & 4 & 4.3 & 2 & 2 & \\
\hline \multirow[t]{2}{*}{ LNR } & $<0.118$ & 323 & 66.9 & 154 & 169 & 0.039 & 88 & 93.6 & 37 & 51 & 0.398 \\
\hline & $\geq 0.118$ & 160 & 33.1 & 56 & 104 & & 6 & 6.4 & 4 & 2 & \\
\hline \multirow[t]{2}{*}{ Chemotherapy } & No & 253 & 52.4 & 113 & 140 & 0.854 & 32 & 34.0 & 18 & 14 & 0.084 \\
\hline & Yes & 230 & 47.6 & 100 & 130 & & 62 & 66.0 & 23 & 39 & \\
\hline \multirow[t]{2}{*}{ Radiotherapy } & No & 342 & 70.8 & 148 & 194 & 0.615 & 66 & 70.2 & 31 & 35 & 0.368 \\
\hline & Yes & 141 & 29.2 & 65 & 76 & & 28 & 29.8 & 10 & 18 & \\
\hline \multirow[t]{5}{*}{ TNM 8th stage } & $\mid A$ & 46 & 9.5 & 25 & 21 & 0.067 & 11 & 11.6 & 1 & 10 & 0.191 \\
\hline & $\mathrm{IB}$ & 68 & 14.1 & 31 & 37 & & 24 & 25.5 & 12 & 12 & \\
\hline & $\| A$ & 58 & 12.0 & 31 & 27 & & 51 & 54.3 & 24 & 27 & \\
\hline & $\| \mathrm{B}$ & 143 & 29.6 & 61 & 82 & & 4 & 4.3 & 2 & 2 & \\
\hline & III & 168 & 34.8 & 73 & 95 & & 4 & 4.3 & 2 & 2 & \\
\hline
\end{tabular}

LN, lymph node; LNR, lymph node ratio; TNM, tumor-node-metastasis. 
Computing, Vienna, Austria. http://www.r-project.org). A twotailed $P$-value was considered statistically significant if $<0.05$.

\section{RESULTS}

\section{Patient Characteristics}

The work flow of the current study is shown in Figure 1. The data of a total of 483 eligible patients with PDAC of the body and tail after surgery were obtained from the SEER database. There were 213 patients with PDAC of the body, and the other 270 patients had PDAC of the tail. A summary of baseline characteristics of the patients is shown in Table 1. The median age of patients in the SEER database was 65 years (range: 32-91 years). Of these patients, $238(49.3 \%)$ were male. A tumor size larger than $4 \mathrm{~cm}(222 ; 46.0 \%)$ was the most common size. Moderately differentiated tumors $(242 ; 50.1 \%)$ were most common, followed by poorly differentiated $(194 ; 40.2 \%)$ and well-differentiated tumors $(47 ; 9.7 \%)$. A total of $252(52.2 \%)$ patients had LN metastasis. Most patients $(168 ; 34.8 \%)$ were categorized as TNM stage III, $29.6 \%$ (143) were stage IIB, $12.0 \%$ (58) were stage IIA, and $23.6 \%$ (114) were stage I. In addition, the proportions of patients with LN metastasis and higher LNR values were higher in patients with PDAC of the tail. Other than these two variables, the other characteristics, including age, gender, race,
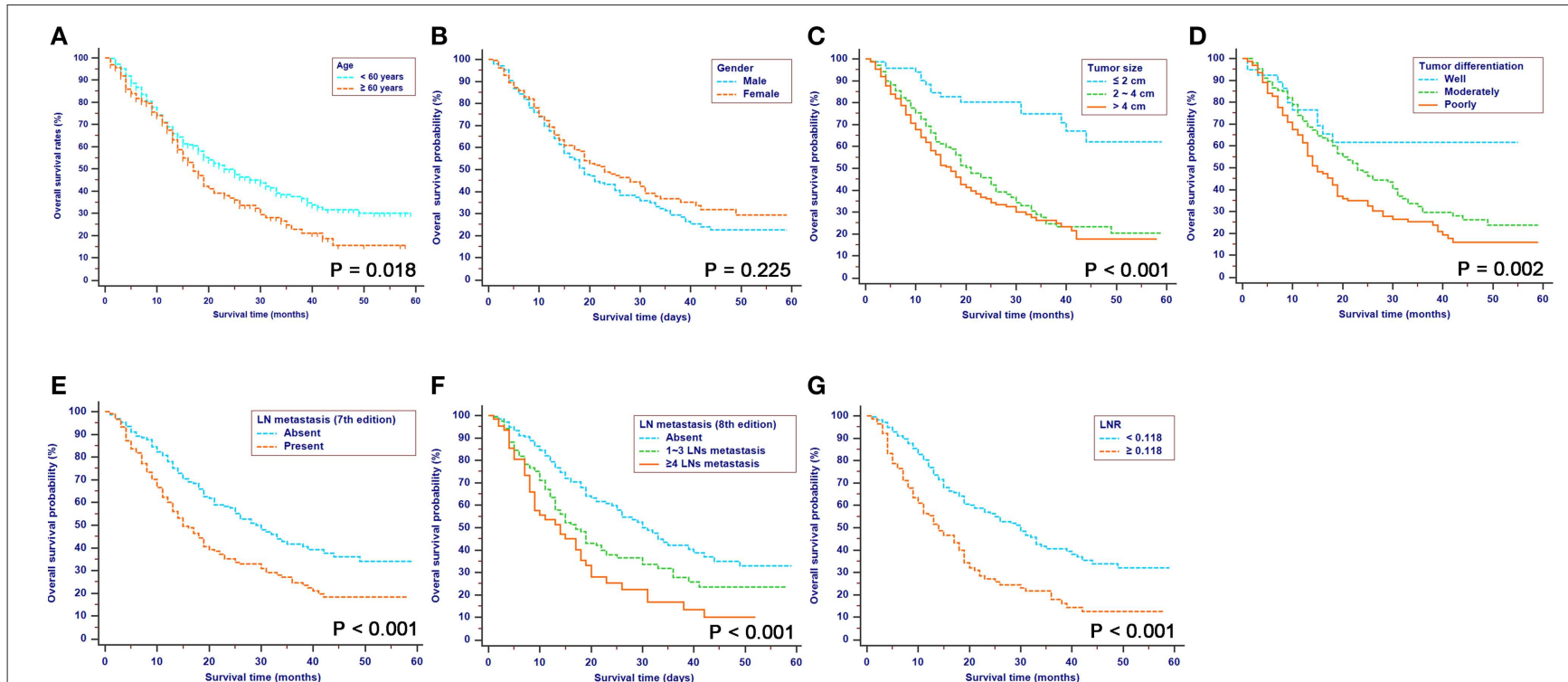

FIGURE 2 | Kaplan-Meier OS curves stratified by patient characteristics: (A) Age; (B) Gender; (C) Tumor size; (D) Tumor differentiation; (E) LN metastasis (7th edition); (F) LN metastasis (8th edition); (G) LNR. OS, overall survival; LN, lymph node; LNR, lymph node ratio; TNM, Tumor-Node-Metastasis.

TABLE 2 | Univariate and multivariate analyses of survival in patients with pancreatic adenocarcinoma of the body and tail.

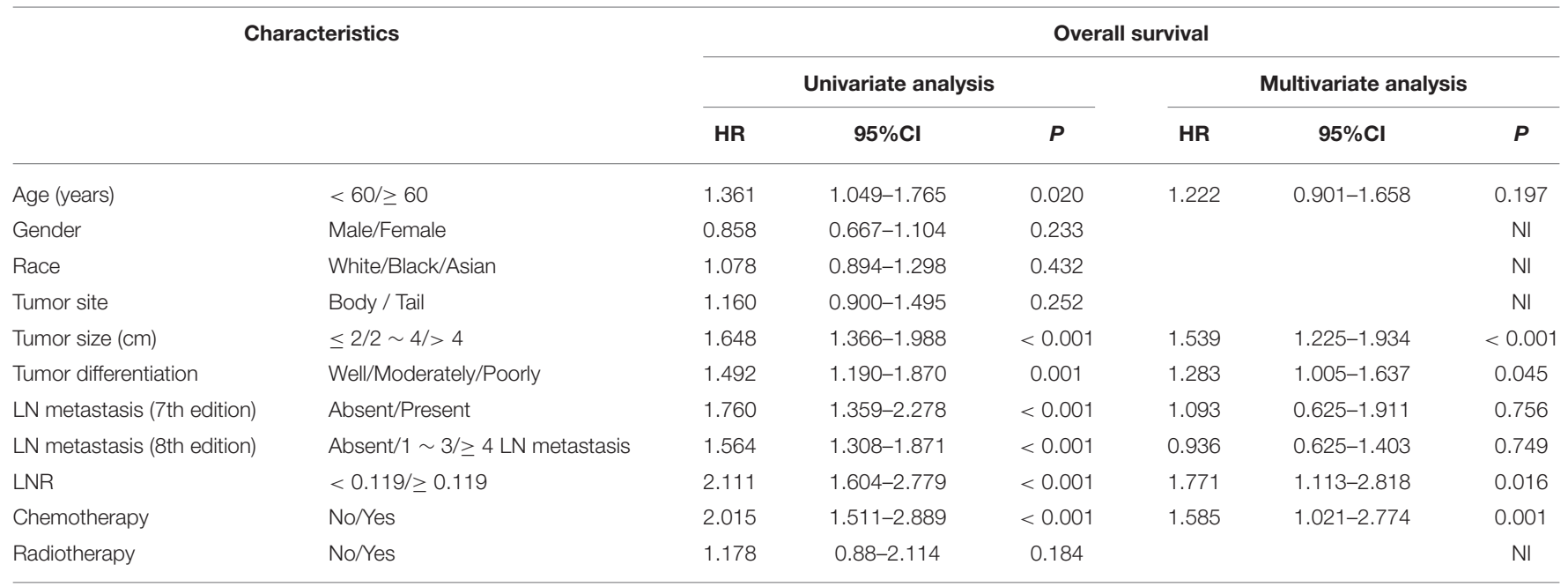

HR, hazard ratio; Cl, confidence interval; NI, not included. 
tumor size, tumor differentiation, and TNM stage (8th edition), were comparable. LNR, with a cutoff value of 0.118 , showed the greatest predictive power and was used to analyze survival in this study (Supplementary Figure 1). A total of 230 (47.6\%) patients had received chemotherapy while $141(29.2 \%)$ patients received radiotherapy in this study.

Additionally, 94 patients from the SYSUCC cohort were included. Similar to patients from the SEER cohort, most patients were younger than 60 years old. Cases that had a moderately-differentiated tumor or absence of LN metastasis, made up most of the patients and more than half of patients had tumors that were larger than $4 \mathrm{~cm}$. A total of $62(66.0 \%)$ patients had received chemotherapy while 28 (29.8\%) patients received radiotherapy in this study. The two groups of patients had substantially balanced clinical and pathological variables.

\section{OS Analysis}

For patients in the SEER cohort, the median OS was 20 months, and the 1-, 2-, and 3-year OS rates were 68.4, 45.7 , and $32.8 \%$, respectively. Patients were stratified by characteristics for the OS analyses. The differences in OS rates were all significant, except upon stratification by gender and race (Figure 2 and Table 1). As shown in Table 2, gender, race, and tumor site were not risk factors for OS $(p>0.050)$, while age, tumor size and differentiation, LN metastasis (7th edition), LN metastasis (8th edition), LNR, chemotherapy, and radiotherapy were significantly associated with OS in the univariate analysis. The multivariate analysis, which incorporated these variables, indicated that tumor size, tumor differentiation and LNR sustained their significance in terms of OS after adjusting for covariates. In addition, older patients had a trend for poor OS compared with younger patients,

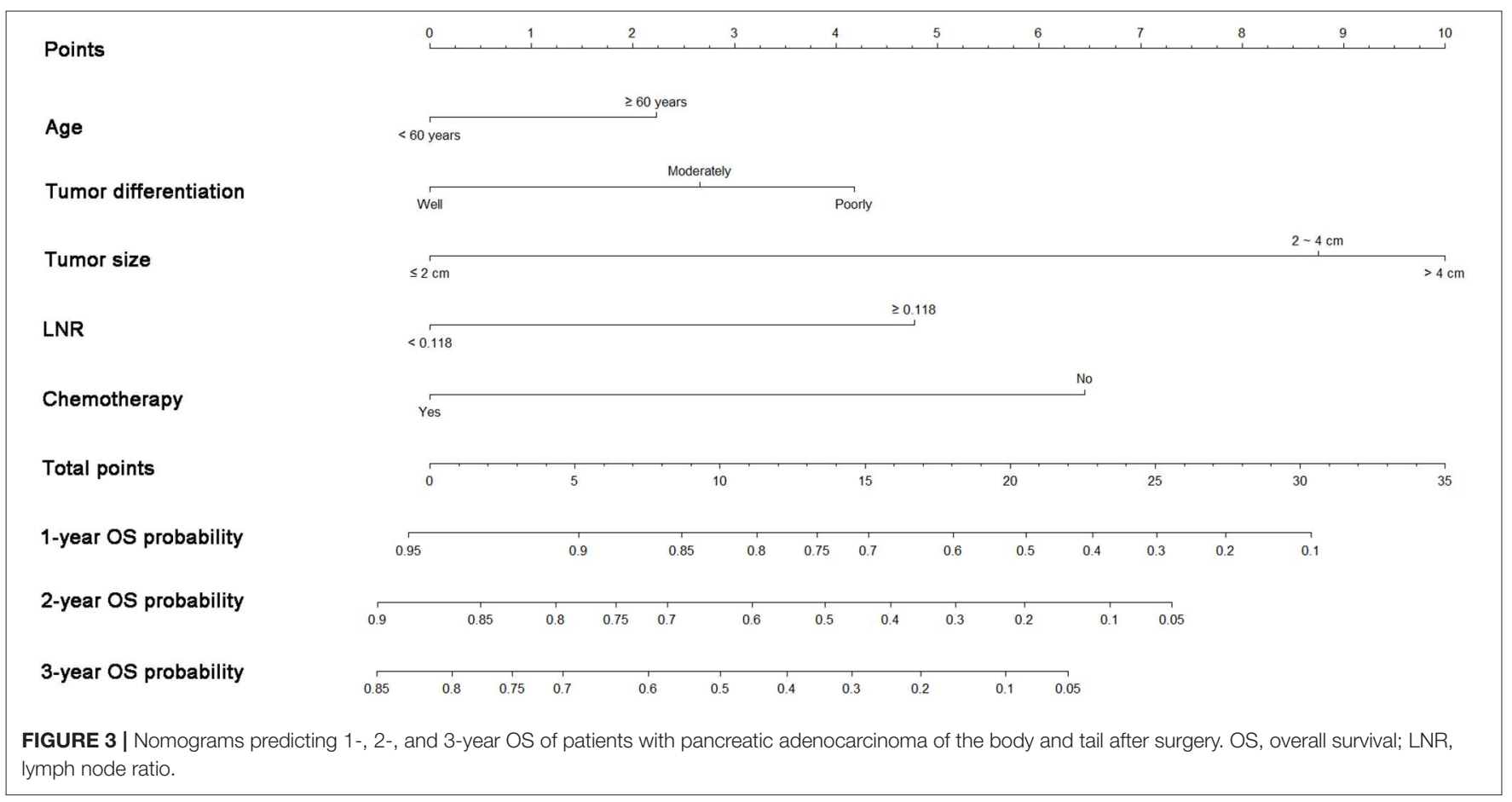

TABLE 3 | Comparison of the C-index and AUC values between nomograms and TNM stages.

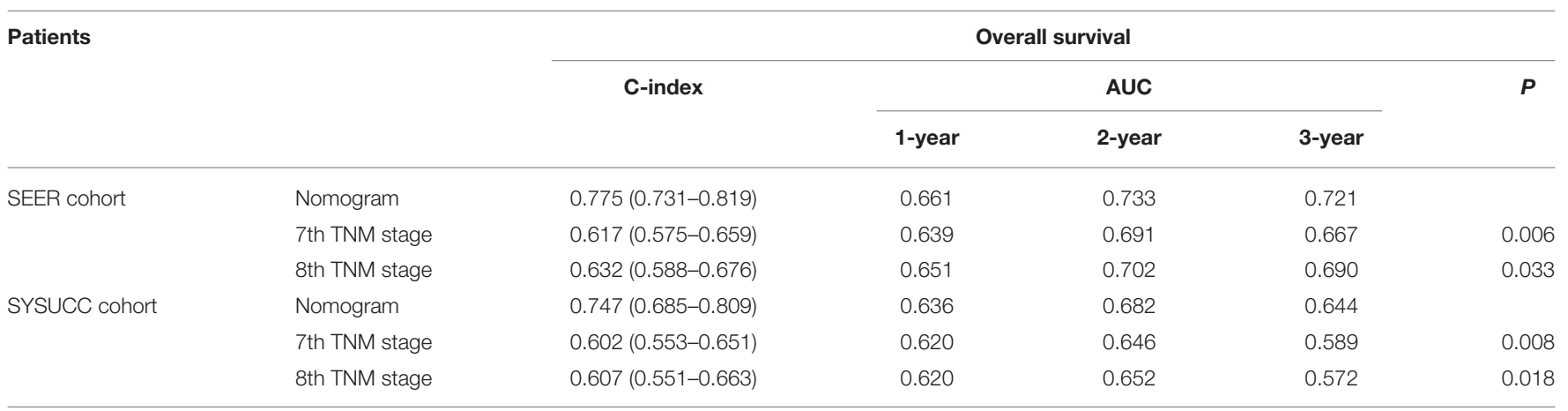

C-index, concordance index; AUC, area under receiver operating characteristic curves. 
while the multivariate analysis failed to show age as a significant predictor of OS.

\section{Construction of the Nomogram}

A nomogram was built using the abovementioned variables via the Cox proportional hazards model. As shown in Figure 3, the nomogram predicted the OS probabilities of 1-3 years for patients with PDAC of the body and tail after surgery. For patients from the SEER cohort, the bias-corrected concordance indexes (C-indexes) of the nomogram were higher than those of the 7th edition of the TNM staging system [0.775 (95\% CI, 0.7310.819 ) vs. 0.617 (95\% CI, $0.575-0.659), P=0.006]$ and the 8 th edition of the TNM staging system [0.775 (95\% CI, 0.731-0.819) vs. 0.632 (95\% CI, 0.588-0.676), $P=0.033$ ]. Elevated C-indexes of the established nomogram were also observed in patients from the SYSUCC cohort, compared with the TNM staging system (Table 3). Excellent agreement between the predictive and the actual observed 1-, 2-, and 3-year OS survival was shown by calibration plots (Figure 4). The discriminatory power of the newly developed nomogram was superior to that of the TNM staging system in this study.

\section{Comparison of AUC Values of the Nomogram and TNM Staging System}

The ROC curves were used to compare the precision of the 1-, 2-, and 3 -year OS predictions. For the training cohort from the SEER database, the AUC values of the nomogram that predicted 1-, 2, and 3-year OS rates were $0.661,0.733$, and 0.721 , respectively, whereas the values of the TNM staging system were $0.639,0.691$, and 0.667 ( 7 th edition) and $0.651,0.702$, and 0.690 (8th edition), respectively. In patients from the external validation cohort, the established nomogram also displayed significantly higher values of AUC than those of the TNM stage system (Table 3). As shown in Figure 5, the nomogram exhibited superior predictive ability to that of the TNM staging system.

\section{DISCUSSION}

The differences in characteristics and prognoses between PDAC of the head and those of the body and tail urged us to pursue a specific staging system for pancreatic body and tail cancer, even though previous studies have compared the predictive power of nomograms in terms of PDAC, $(21,22)$ which were primarily focused on pancreatic head cancer. In the present study, using a relatively large cohort, a novel nomogram was established to predict OS rates in patients with PDAC of the body and tail in this study. The well-established nomogram seemed to be more significantly predictive than the TNM staging system.

The assessment and prediction of prognosis by the established nomogram differed from those made by current TNM staging systems. Tumor differentiation was proven to be an independent prognostic factor for predicting OS in these patients and

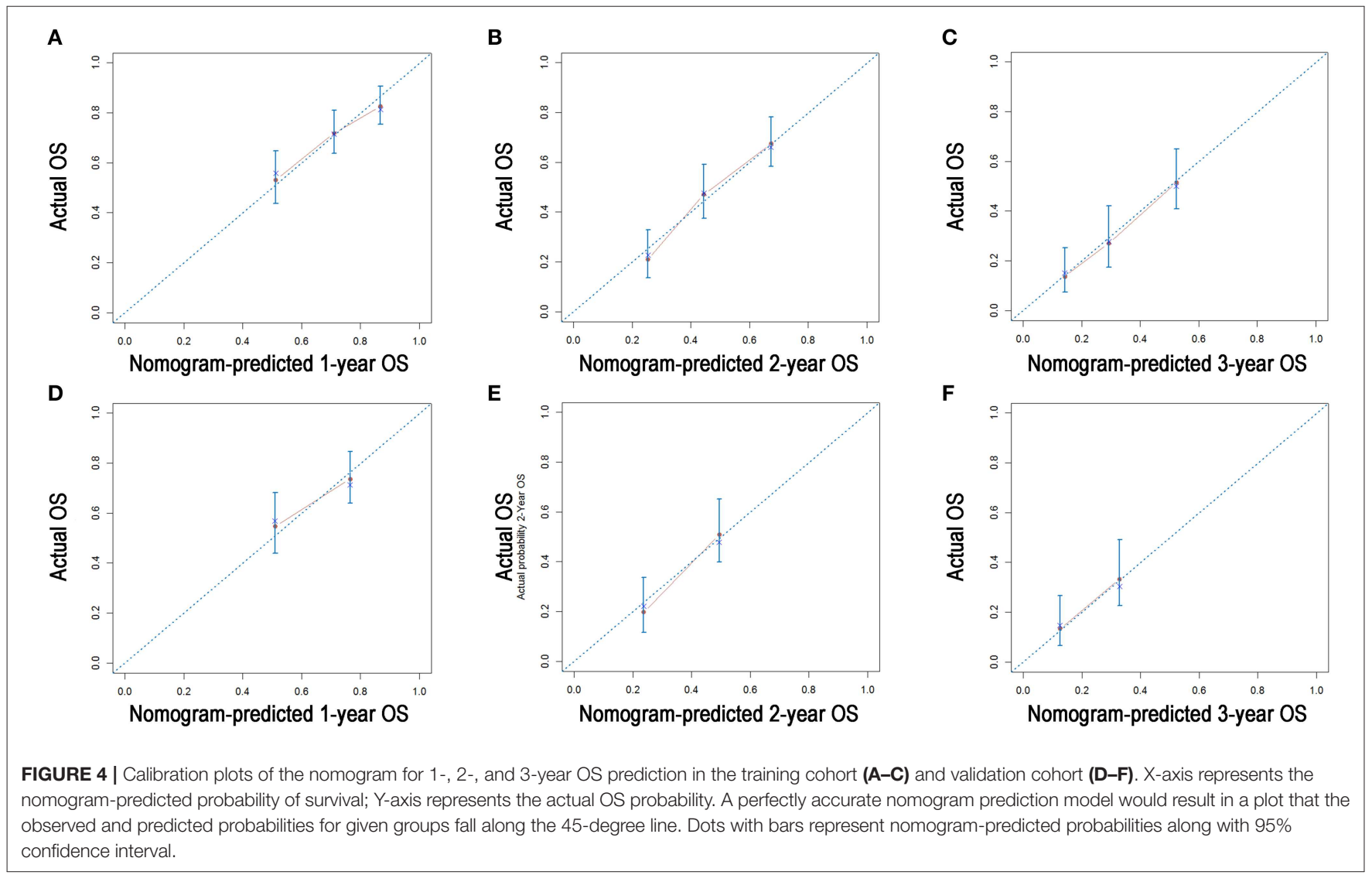




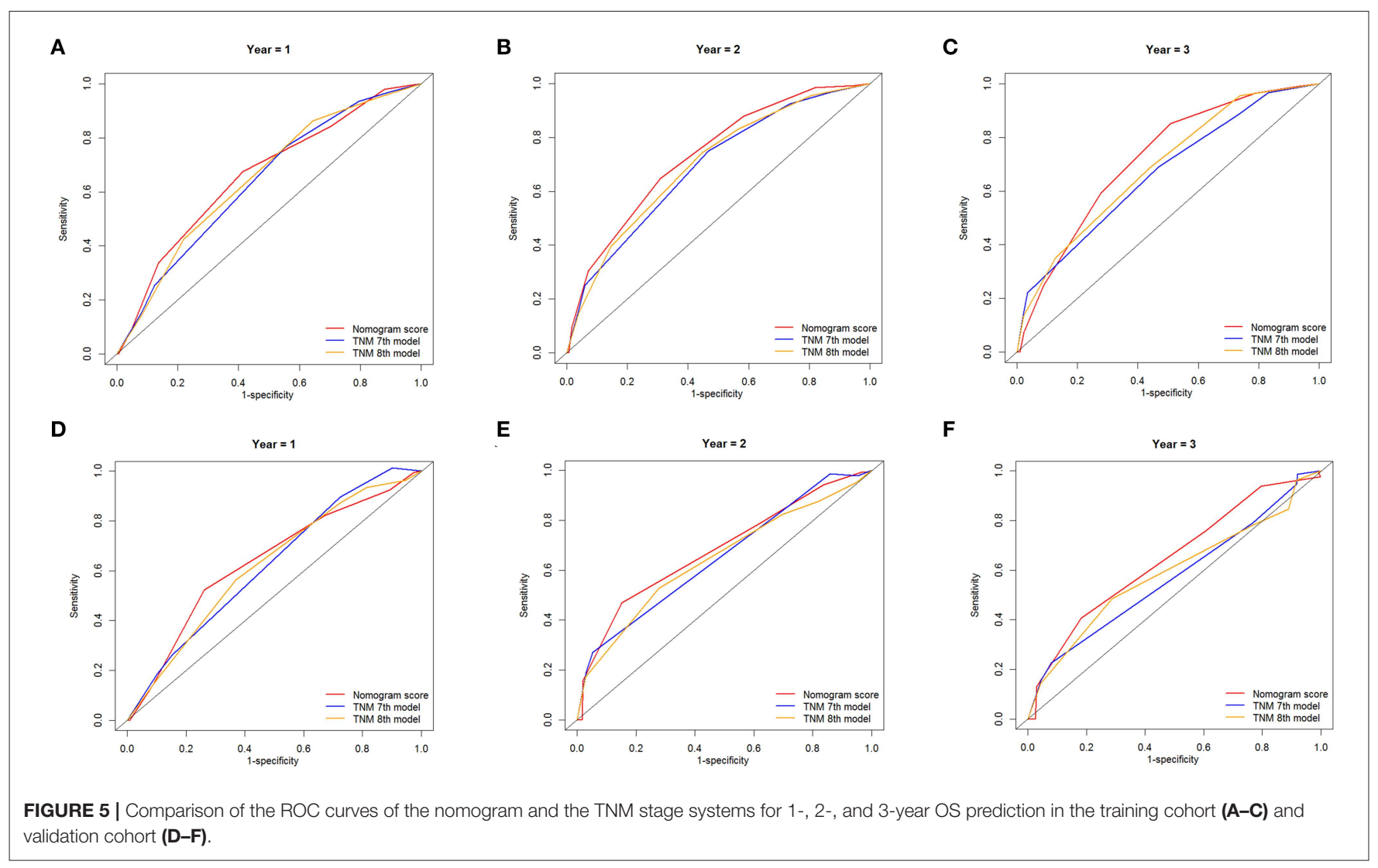

was incorporated into the nomogram. Previous studies have confirmed the prognostic significance of tumor differentiation in PDAC, $(23,24)$ and this was consistent with our results. As a pathological variable which was independent of other variables, including tumor size and LN metastasis, may provide additive prognostic power in survival estimation. According to the nomogram, different levels of tumor differentiation meant different points and different OS rates in patients, even if they had tumors with the same TNM stage. The additive predictive power provided by these variables surely contributed to the superior power of the nomogram for predicting OS, compared with the 7 th and 8th editions of the TNM staging system.

Moreover, patients stratified by age had significantly different OS rates in this study. Age alone appeared to affect survival rates after surgical resection in patients with pancreatic cancer (25). Age-related comorbid conditions or complications could possibly contribute to mortality in older patients. Age failed to be classified as an independent prognostic factor, although significant differences in OS were observed among patients stratified by age in this study. It is possible that an even larger cohort will confirm the prognostic significance of age in survival analyses. It was expected that age would have an important role in predicting survival (26). Thus, age was added to the nomogram established in this study and was capable of improving the prognostic efficiency.

Currently, LN involvement remains one of the most important predictors of survival in patients with $\operatorname{PDAC}(27,28)$.
An interesting phenomenon was shown in this study: the classic "N" stages of both the 7th and 8th editions of the TNM staging system failed to exhibit independent predictive significance, whereas LNR was proven to be an independent predictive factor in the OS analysis; these results were similar to those of other studies $(29,30)$. The time-dependent ROC curve analysis was used to determine the optimal cutoff value for LNR. Similar to the results of Pan et al. (31) this method, which was used in many similar reports, (32-34) decided the cutoff value of LNR with more predictive power due to the consideration of the survival time in evaluating the values. As a significant modifier of the effects of LN status, (35) LNR showed the potential for LN metastasis, which was similar with previous studies (36). In this sense, LNR was established as a better predictor of OS than the status of LN metastasis, (37) and a higher LNR value was strongly associated with poor distant metastasis-free survival (38).

In addition to the abovementioned factors, tumor size and chemotherapy were also identified as significant predictors of survival in these patients. Consistent with many previous reports, $(21,39)$ our results confirmed the significant predictive roles of these two factors in predicting survival in patients with PDAC. Moreover, previous studies had found that status of resection margin of surgery for PDAC of the body and tail had great impact on survival. For a more accurate estimation of survival, all patients included in this study had received radical resection and the impact of resection margin on survival was minimal in this study. Thus, in the nomogram model, 
each factor from the multivariate Cox proportional hazard regression model was ascribed a weighted point total that implied a survival prognosis. The established nomogram makes it easier for physicians to assess a variety of parameters with more objectivity and precision and to distinguish subgroups with different prognoses among patients with PDAC of the body and tail after radical resection. There are two major applications of the nomogram. First, as a quantitative scoring system, the nomogram can be used to predict survival of patients with PDAC of the body and tail. Second, for patients with high scores calculated by the established nomogram, close follow-up or appropriate treatment may be required. The combination of the main elements from the TNM staging system and other tumor-associated indices, including age, tumor differentiation and LNR, would surely contribute to a better discriminatory power of the nomogram in predicting survival, compared with the TNM staging system. The established nomogram can be used as a practical tool to predict clinical outcomes, and it has potential use in decision-making regarding subsequent treatment of patients with PDAC of the body and tail after surgery.

There are limitations in the present study that should be addressed. First, this was a retrospective study that relied on the SEER database. Some of the potential predictors of survival, such as perineural invasion, lymphovascular invasion, and portal vein involvement, could not be included in the nomogram. Second, although good fitness was demonstrated for the validation in the present study, we should recognize that bootstrapping is only helpful to reduce the overfit bias of the nomogram. More validations using large and independent cohorts are necessary for the present nomogram.

In this study, we analyzed the prognostic data of PDAC of the body and tail using the SEER database. A nomogram for the estimation of 1-, 2-, and 3-year OS was established based on a large study cohort for the first time. The present nomogram can predict the prognosis of patients with PDAC of the body and tail

\section{REFERENCES}

1. Siegel RL. Cancer statistics. CA: Cancer J Clin. (2018) 68:730. doi: 10.3322/caac. 21442

2. Salem AI, Alfi M, Winslow E, Cho CS, Weber SM. Has survival following pancreaticoduodenectomy for pancreas adenocarcinoma improved over time? J Surg Oncol. (2015) 112:643-9. doi: 10.1002/jso. 24048

3. Ling Q, Xu X, Zheng SS, Kalthoff $\mathrm{H}$. The diversity between pancreatic head and body/tail cancers: clinical parameters and in vitro models. Hepatobiliary Pancreat Dis Int. (2013) 12:480-7. doi: 10.1016/S1499-3872(13) 60076-4

4. Wagner M, Redaelli C, Lietz M, Seiler CA, Friess H, Büchler MW. Curative resection is the single most important factor determining outcome in patients with pancreatic adenocarcinoma. Br J Surg. (2004) 91:58694. doi: 10.1002/bjs.4484

5. Paye F, Micelli Lupinacci R, Bachellier P, Boher JM, Delpero JR. Distal pancreatectomy for pancreatic carcinoma in the era of multimodal treatment. Br J Surg. (2015) 102:229-36. doi: 10.1002/bjs.9708

6. Kayahara M, Nagakawa T, Futagami F, Kitagawa H, Ohta T, Miyazaki I. Lymphatic flow and neural plexus invasion associated with carcinoma after surgery with considerable accuracy and can help doctors provide highly tailored patient management in the future.

\section{DATA AVAILABILITY STATEMENT}

The datasets generated for this study are available on request to the corresponding author.

\section{ETHICS STATEMENT}

The studies involving human participants were reviewed and approved by Institutional Review Board (IRB) of the Sun Yatsen University Cancer Center. The patients/participants provided their written informed consent to participate in this study.

\section{AUTHOR CONTRIBUTIONS}

SL designed the project, reviewed and edited the manuscript, respectively. $\mathrm{CH}, \mathrm{SS}$, and $\mathrm{YZ}$ performed the study selection, data extraction, statistical analyses and wrote the main manuscripts. $\mathrm{CH}, \mathrm{SS}, \mathrm{YZ}$, and $\mathrm{XL}$ contributed to the classification criteria discussion. All authors reviewed the manuscript.

\section{FUNDING}

This work was supported by grants from the National Natural Science Funds (No. 81672390) and the National Key Research and Development Plan (No. 2017YFC0910002).

\section{SUPPLEMENTARY MATERIAL}

The Supplementary Material for this article can be found online at: https://www.frontiersin.org/articles/10.3389/fonc. 2020.00590/full\#supplementary-material

Supplementary Figure 1 | The ROC curve of survival based on the changes of LNR value.

of the body and tail of the pancreas. Cancer. (1996) 78:248591. doi: 10.1002/(SICI)1097-0142(19961215)78:12<2485::AID-CNCR6>3.0. CO;2-J

7. Pu N, Lv Y, Zhao G, Lee W, Nuerxiati A, Wang D, et al. Survival prediction in pancreatic cancer patients with no distant metastasis: a large-scale populationbased estimate. Future Oncol. (2018) 14:165-75. doi: 10.2217/fon-2017-0380

8. Sahin TT, Fujii T, Kanda M, Nagai S, Kodera Y, Kanzaki A, et al. Prognostic implications of lymph node metastases in carcinoma of the body and tail of the pancreas. Pancreas. (2011) 40:1029-33. doi: 10.1097/MPA.0b013e3182207893

9. Amin MB ES, Greene F. AJCC Cancer Staging Manual, 8th ed. Chicago, IL: Springer (2017).

10. Rauscher I, Düwel C, Haller B, Rischpler C, Heck MM, Gschwend JE, et al. Efficacy, predictive factors, and prediction nomograms for (68)Ga-labeled prostate-specific membrane antigen-ligand positronemission tomography/computed tomography in early biochemical recurrent prostate cancer after radical prostatectomy. Eur Urol. (2018) 73:656-61. doi: 10.1016/j.eururo.2018.01.006

11. Sjoquist KM, Renfro LA, Simes RJ, Tebbutt NC, Clarke S, Seymour MT, et al. Personalizing survival predictions in advanced colorectal cancer: The ARCAD nomogram project. J Natl Cancer Inst. (2017) 110:63848. doi: $10.1093 /$ jnci/djx 253 
12. Fakhry C, Zhang Q, Nguyen-Tân PF, Rosenthal DI, Weber RS, Lambert L, et al. Development and validation of nomograms predictive of overall and progression-free survival in patients with oropharyngeal cancer. J Clin Oncol. (2017) 35:4057-65. doi: 10.1200/JCO.2016.72.0748

13. Battersby NJ, Bouliotis G, Emmertsen KJ, Juul T, Glynne-Jones R, Branagan $\mathrm{G}$, et al. Development and external validation of a nomogram and online tool to predict bowel dysfunction following restorative rectal cancer resection: the POLARS score. Gut. (2017) 64:688-96. doi: 10.1136/gutjnl-2016-312695

14. Sternberg $\mathrm{CN}$. Are nomograms better than currently available stage groupings for bladder cancer? J Clinical Oncol. (2006) 24:3819-20. doi: 10.1200/JCO.2006.07.1290

15. Fu YP, Ni XC, Yi Y, Cai XY, He HW, Wang JX, et al. A novel and validated Inflammation-Based Score (IBS) predicts survival in patients with hepatocellular carcinoma following curative surgical resection: a STROBE-compliant article. Medicine. (2016) 95:e2784. doi: 10.1097/MD.0000000000002784

16. Lu J, Zheng ZF, Xie JW, Wang JB, Lin JX, Chen QY, et al. Is the 8th Edition of the AJCC TNM staging system sufficiently reasonable for all patients with noncardia gastric cancer? A 12,549-patient international database study. Ann Surg Oncol. (2018) 25:2002-11. doi: 10.1245/s10434-018-6447-0

17. Lu J, Zheng ZF, Wang W, Xie JW, Wang JB, Lin JX, et al. A novel TNM staging system for gastric cancer based on the metro-ticket paradigm: a comparative study with the AJCC-TNM staging system. Gastric Cancer. (2019) 22:75968. doi: 10.1007/s10120-018-00904-w

18. He C, Zhang Y, Cai Z, Lin X. Competing risk analyses of overall survival and cancer-specific survival in patients with combined hepatocellular cholangiocarcinoma after surgery. BMC Cancer. (2019) 19:178. doi: 10.1186/s12885-019-5398-6

19. Harrell FE Jr, Lee KL, Mark DB. Multivariable prognostic models: issues in developing models, evaluating assumptions and adequacy, and measuring and reducing errors. Stat Med. (1996) 15:361-87.

20. Pencina MJ, D'Agostino RB. Overall $\mathrm{C}$ as a measure of discrimination in survival analysis: model specific population value and confidence interval estimation. Stat Med. (2004) 23:2109-23. doi: 10.1002/sim.1802

21. Deng QL, Dong S, Wang L, Zhang CY, Ying HF, Li ZS, et al. Development and validation of a nomogram for predicting survival in patients with advanced pancreatic ductal adenocarcinoma. Sci Rep. (2017) 7:11524. doi: 10.1038/s41598-017-11227-8

22. Xu J, Shi KQ, Chen BC, Huang ZP, Lu FY, Zhou MT. A nomogram based on preoperative inflammatory markers predicting the overall survival of pancreatic ductal adenocarcinoma. J Gastroenterol Hepatol. (2017) 32:1394402. doi: 10.1111/jgh.13676

23. Hornick JR, Johnston FM, Simon PO, Younkin M, Chamberlin M, Mitchem JB, et al. A single-institution review of 157 patients presenting with benign and malignant tumors of the ampulla of vater: management and outcomes. Surgery. (2011) 150:169-76. doi: 10.1016/j.surg.2011.05.012

24. Winter JM, Cameron JL, Olino K, Herman JM, de Jong MC, Hruban RH, et al. Clinicopathologic analysis of ampullary neoplasms in 450 patients: implications for surgical strategy and long-term prognosis. J Gastrointest Surg. (2010) 14:379-87. doi: 10.1007/s11605-009-1080-7

25. Eubanks A, Pepe J, Veldhuis P, de la Fuente SG. Age as a prognostic indicator for adjuvant therapy in patients who underwent pancreatic resections for cancer. J Geriatr Oncol. (2018) 9:362-66. doi: 10.1016/j.jgo.2017.12.004

26. Zhou H, Zhang Y, Qiu Z, Chen G, Hong S, Chen X, et al. Nomogram to predict cause-specific mortality in patients with surgically resected stage i nonsmall-cell lung cancer: a competing risk analysis. Clin Lung Cancer. (2017) 19:e195-203. doi: 10.1016/j.cllc.2017.10.016

27. Valsangkar NP, Bush DM, Michaelson JS, Ferrone CR, Wargo JA, Lillemoe KD, et al. N0/N1, PNL, or LNR? The effect of lymph node number on accurate survival prediction in pancreatic ductal adenocarcinoma. J Geriatr Oncol. (2013) 17:257-66. doi: 10.1007/s11605-012-1974-7

28. Zhan HX, Xu JW, Wang L, Zhang GY, Hu SY. Lymph node ratio is an independent prognostic factor for patients after resection of pancreatic cancer. J Surg Oncol. (2015) 13:105. doi: 10.1186/s12957-015-0510-0

29. Yamada S, Fujii T, Hirakawa A, Kanda M, Sugimoto H, Kodera Y. Lymph node ratio as parameter of regional lymph node involvement in pancreatic cancer. Langenbecks Arch Surg. (2016) 401:1143-52. doi: 10.1007/s00423-016-1412-5

30. Dasari BV, Roberts KJ, Hodson J, Stevens L, Smith AM, Hubscher SG et al. A model to predict survival following pancreaticoduodenectomy for malignancy based on tumour site, stage and lymph node ratio. HPB. (2016) 18:332-8. doi: 10.1016/j.hpb.2015.11.008

31. Pan Z, Bu Q, You H, Yang J, Liu Q, Lyu J. Determining the optimal cutoff point for lymph node density and its impact on overall survival in children with Wilms' tumor. Cancer Manag Res. (2019) 11:75966. doi: 10.2147/CMAR.S190138

32. Tada T, Kumada T, Toyoda H, Kiriyama S, Tanikawa M, Hisanaga Y, et al. HBcrAg predicts hepatocellular carcinoma development: An analysis using time-dependent receiver operating characteristics. J Hepatol. (2016) 65:4856. doi: 10.1016/j.jhep.2016.03.013

33. He C, Huang $\mathrm{X}$, Zhang $\mathrm{Y}$, Cai Z, Lin $\mathrm{X}$, Li S. A quantitative clinicopathological signature for predicting recurrence risk of pancreatic ductal adenocarcinoma after radical resection. Front Oncol. (2019) 9:1197. doi: 10.3389/fonc.2019.01197

34. Ross AE, Yousefi K, Davicioni E, Ghadessi M, Johnson MH, Sundi D, et al. Utility of risk models in decision making after radical prostatectomy: lessons from a natural history cohort of intermediate- and high-risk men. Eur Urol. (2016) 69:496-504. doi: 10.1016/j.eururo.2015.04.016

35. Smith BJ, Mezhir JJ. An interactive Bayesian model for prediction of lymph node ratio and survival in pancreatic cancer patients. JAMIA. (2014) 21:e20311. doi: 10.1136/amiajnl-2013-002171

36. Ashfaq A, Pockaj BA, Gray RJ, Halfdanarson TR, Wasif N. Nodal counts and lymph node ratio impact survival after distal pancreatectomy for pancreatic adenocarcinoma. J Gastrointest Surg. (2014) 18:192935. doi: 10.1007/s11605-014-2566-5

37. Tol JA, Brosens LA, van Dieren S, van Gulik TM, Busch OR, Besselink MG, et al. Impact of lymph node ratio on survival in patients with pancreatic and periampullary cancer. $\mathrm{Br}$ J Surg. (2015) 102:237-45. doi: 10.1002/ bjs.9709

38. Roland CL, Katz MH, Gonzalez GM, Pisters PW, Vauthey JN, Wolff RA, et al. A high positive lymph node ratio is associated with distant recurrence after surgical resection of ampullary carcinoma. J Gastrointest Surg. (2012) 16:2056-63. doi: 10.1007/s11605-012-2015-2

39. Hamada T, Nakai Y, Yasunaga $H$, Isayama $H$, Matsui $H$, Takahara $N$, et al. Prognostic nomogram for nonresectable pancreatic cancer treated with gemcitabine-based chemotherapy. Br J Cancer. (2014) 110:19439. doi: 10.1038/bjc.2014.131

Conflict of Interest: The authors declare that the research was conducted in the absence of any commercial or financial relationships that could be construed as a potential conflict of interest.

Copyright (c) $2020 \mathrm{He}$, Sun, Zhang, Lin and Li. This is an open-access article distributed under the terms of the Creative Commons Attribution License (CC BY). The use, distribution or reproduction in other forums is permitted, provided the original author(s) and the copyright owner(s) are credited and that the original publication in this journal is cited, in accordance with accepted academic practice. No use, distribution or reproduction is permitted which does not comply with these terms. 